\title{
Serological and Genetic Characterization of a Human Monoclonal Immunoglobulin G Anti-DNA Idiotype
}

Michael R. Ehrenstein, * Celia M. Longhurst, ${ }^{\star *}$ David S. Latchman, ${ }^{\star}$ and David A. Isenberg ${ }^{*}$

Divisions of ${ }^{*}$ Rheumatology and ${ }^{\ddagger}$ Molecular Pathology, University College London Medical School, London W1P 9PG, United Kingdom

\begin{abstract}
This study analyzed the distribution of an idiotype, B3-Id, in patients with active SLE, classified according to organ involvement, normal controls, and other autoimmune rheumatic diseases. A polyclonal anti-idiotype was raised by immunizing a rabbit with a monoclonal IgG anti-double-stranded (ds) DNA antibody, B3, generated from a patient with SLE who had active arthritis. The idiotype is present on the $\lambda$ chain and is at or near the binding site for double-stranded DNA. The $\lambda$ chain, which was characterized by nucleotide sequencing, was $90 \%$ homologous to the V $\lambda 2.1$ germline, which is known to be involved in coding for nephritogenic anti-DNA antibodies carrying the 8.12 idiotype. There were four changes to positively charged amino acids, known to be involved in DNA binding, in the complementarity determining regions of $\mathrm{B} 3 \lambda$ chain compared with a non-DNA binding, 8.12 positive antibody, PV11. Only one change to a positively charged amino acid occurs in the heavy chain of B3, which is $93.5 \%$ homologous to VH-26.

The B3-Id was present on IgG antibodies in the serum of $20 \%$ of patients with SLE but was not found in the normal controls. Within the SLE group, there is a statistically significant association of B3-Id on IgG in the arthritis group (42\%) compared to the other manifestations $(9 \%)(P<0.001)$. In four B3-Id-positive SLE patients tested serially, the level of B3-Id reflected the arthritis disease activity more closely than the overall disease activity $(P<0.05)$. The B3-Id was also present on IgM antibodies in one third of patients with rheumatoid arthritis.

This idiotype is the first to be derived from a human monoclonal anti-DNA antibody of the IgG class, the isotype associated with active disease. Sequence analysis shows that positively charged amino acids on the $\lambda$ chain may contribute to DNA binding. (J. Clin. Invest. 1994. 93:1787-1797.) Key words: systemic lupus erythematosus - immunoglobulin $\mathbf{G} \bullet$ monoclonal $\bullet$ idiotype $\bullet$ arthritis
\end{abstract}

\section{Introduction}

Immunoglobulin idiotypes are phenotypic markers of the variable genes used to encode immunoglobulin molecules. They

Address correspondence to Dr. M. Ehrenstein, Department of Rheumatology Research, University College, London Medical School, Arthur Stanley House, 40-50 Tottenham Street, London W1P 9PG, United Kingdom.

Received for publication 7 July 1993 and in revised form 22 November 1993.

J. Clin. Invest.

(c) The American Society for Clinical Investigation, Inc.

$0021-9738 / 94 / 04 / 1787 / 11 \quad \$ 2.00$

Volume 93, May 1994, 1787-1797 are identified by anti-idiotypic antibodies. In the past decade, more than 20 apparently different DNA antibody idiotypes have been identified ( reviewed in reference 1), and there have been several attempts to correlate those present in the serum of SLE patients with different serological or clinical manifestations $(1,2)$. Idiotype analysis has been based on three different methodologies. The first has utilized anti-idiotypic antibodies to idiotypes first identified on IgM human monoclonal antiDNA antibodies that may not represent the pathogenic antibodies present in SLE. The second method has been to extract DNA binding antibodies from the sera and to use this heterogeneous material to produce anti-idiotypes. If this latter approach is used, analysis of the idiotype structure involves generation of monoclonal antibodies bearing the particular idiotype and may identify idiotypes that are not confined to one variable gene (3). The third method involves identifying idiotypes on mouse anti-DNA antibodies which are then found to be expressed on human antibodies (4).

Whichever method has been used few of the DNA antibody idiotypes have shown a clear cut association with disease activity. Among those whose levels did appear to fluctuate in this manner were the $16 / 6$ and GN-2 idiotypes and the 9G4 idiotope $(2,5)$. Lack of correlation between levels of idiotypes with different disease manifestations seen in SLE may be caused by the tendency of the investigators to use global score indices. Apart from problems of disease activity index validation, use of these indices may conceal important disease correlations within individual systems. Moreover, it is possible that different mechanisms are responsible for the activity in different organs or systems. In this study, we have utilized the British Isles Lupus Assessment Group (BILAG) ${ }^{1}$ activity index that has been developed to overcome these problems (6) and thus enables the presence of anti-DNA idiotypes to be related to the full range of individual disease manifestations.

The analysis of DNA antibody idiotypes has not always differentiated whether the idiotype is present on the IgG or IgM population. This is a potentially important distinction to make, since idiotype regulation of IgM antibodies may be different from their IgG counterparts, which are more likely to be pathogenic, at least in lupus nephritis (7). Moreover, the presence of an idiotype exclusively on the IgG population may indicate an idiotype generated by somatic mutation.

We have reported the production of a monoclonal IgG antiDNA antibody, B3, from a patient with active SLE (8). This antibody has been used to generate an anti-idiotypic antibody

\footnotetext{
1. Abbreviations used in this paper: BILAG, British Isles Lupus Assessment Group; CNS, central nervous system; CVS/resp, cardiovascular/ respiratory; dsDNA, double-stranded DNA; F/M, female/male; Id, idiotype; ssDNA, single-stranded DNA.
} 
that recognizes a common idiotype (Id) designated B3-Id. We now present the serologic and genetic characterization of B3-Id.

\section{Methods}

101 patients with SLE were studied (mean age $=43 \pm 11.6$; female/ male $(F / M)=92: 3)$. Each of the SLE patients met the American Rheumatism Association's revised criteria for the classification of the disease (9). Disease activity was assessed by the BILAG activity index (6). This index, based on the principle of the physician's intention to treat the disease, assesses the activity of the disease in eight major organs or systems (10). From this assessment, scores are generated ranging from $A$ (most active) to $E$ (no activity in that particular organ system). Only sera taken from patients when their disease was active (i.e., at least one A or B score) were studied (except for serial studies and when the B3-Id level was high, see Results). Although not designed for this purpose, the BILAG index can be adapted to provide a global score, $\mathrm{A}=9$ points, $\mathrm{B}=3, \mathrm{C}=1, \mathrm{D}=0$, and $\mathrm{E}=0$, which has been validated against other global scores (11). The sera studied were taken from patients with active disease on a particular date in one of six systems; i.e., musculoskeletal (M/S), skin, renal, central nervous system (CNS), cardiovascular/respiratory (CVS/resp), and vascular. (A score of A in the musculoskeletal system implies either definite myositis or severe polyarthritis with loss of function only, whereas a score of $B$ implies either definite synovitis or tendonitis only). There was considerable overlap in the BILAG scores between general and hematology and the other systems; there was no bias towards one particular system. Our intention was to analyze dates when only one system was active. Thus, if on one particular date both M/S and CNS systems were active, this sample was excluded. In addition, a number of serum samples from idiotype-positive patients were studied when the disease was inactive and serial bleeds were assessed (see Results). The sera from 27 patients with rheumatoid arthritis (each of whom met the revised criteria of the American Rheumatism Association [12]) (mean age $=57 \pm 13.4 ; \mathrm{F} / \mathrm{M}=14: 13), 20$ patients with primary Sjögren's syndrome (who fulfilled criteria for the disease [13]) (mean age $=58 \pm 14.5 ; \mathrm{F} / \mathrm{M}=15: 5), 17$ patients with idiopathic myositis (14) (mean age $=46 \pm 14.5 ; \mathrm{F}: \mathrm{M} 12: 5$ ), and 28 normal controls (mean age $=39 \pm 11 ; \mathrm{F} / \mathrm{M}=19: 9$ ) were also examined.

Monoclonal antibody and production of anti-idiotype. The production, purification and specificity of B3 has been described in detail elsewhere (8). In brief, peripheral blood lymphocytes from a patient with active SLE were fused with the heteromyeloma CB-F7 (kind gift of Siegbert Jahn, Charite Hospital, Berlin). At the time of fusion this patient had severe polyarthritis, fatigue, mild chest pain, periungual erythema, and livedo reticularis. The BILAG scores were as follows: general, B; skin, C; neurological, D; M/S, A; CVS/resp, B; vascular, C; renal, E; haematology, A. B3 binds predominantly to double-stranded (ds) DNA, but also weakly to single-stranded (ss) DNA in solid phase, by ELISA. The antibody also binds to Crithidia. B3 was purified by passing the supernatant (serum free) over a protein $G$ column (Pharmacia fine Chemicals, Piscataway, NJ) and eluting with $0.1 \mathrm{M}$ glycine$\mathrm{HCl}$ buffer, $\mathrm{pH}$ 2.3. Polyclonal anti-idiotype sera were raised by immunization of rabbits with purified $\mathrm{mAb} B 3$ in incomplete Freund's adjuvant. Polyclonal anti-idiotypic serum was rendered idiotypic specific by extensive absorption over a human IgG/IgM-Sepharose 4B (Pharmacia Fine Chemicals) affinity column until no anti-human Ig activity was detectable by an Ig ELISA. The anti-idiotypic serum was further purified to remove irrelevant specificities by purification on a B3 Sepharose column followed by acid elution of bound antibody. Control rabbit immunoglobulin was raised by injecting rabbits with incomplete Freund's adjuvant alone and removing the anti-Fc activity on a human IgG/IgM-Sepharose 4B affinity column until no anti-human Ig activity was detectable by ELISA.

\section{Characterization of anti-Id-B3}

Competitive ELISA for idiotype expression. Using a previously described method (15), rabbit anti-Id-B3 reagent (at a concentration previously determined to give $50 \%$ maximal binding of anti-Id-B3 to $\mathrm{mAb} \mathrm{B} 3$, that is $50 \mathrm{ng} / \mathrm{ml}$ ) was incubated with varying concentrations of $\mathrm{B} 3$, pooled $\mathrm{IgG}$, or a control $\mathrm{mAb}$ IgG for $1 \mathrm{~h}$ at $37^{\circ} \mathrm{C}$. Reaction mixtures were then added to $\mathrm{mAb} B 3$-coated wells $(1 \mu \mathrm{g} / \mathrm{ml})$ for $1 \mathrm{~h}$ at $37^{\circ} \mathrm{C}$. A mouse monoclonal anti-rabbit alkaline phosphatase conjugate (Sigma Immunochemicals, St. Louis, MO) was used to determine the amount of anti-idiotype bound.

Competitive ELISA for antigen binding site idiotype expression. The method has been described (15). Reaction mixtures of mAb at the same concentration and antigen at varying concentrations, in this case, calf thymus dsDNA, ssDNA, and RNA, were incubated for $1 \mathrm{~h}$ at $37^{\circ} \mathrm{C}$ before being transferred to anti-Id-coated wells for $1 \mathrm{~h}$ at $37^{\circ} \mathrm{C}$. The amount of human monoclonal antibody that had bound to the plate was determined by using an anti-human alkaline phosphatase conjugate (Sigma Immunochemicals).

Determination of position of Id on light or heavy chain. To determine whether the B3-Id, as defined by the rabbit anti-B3-Id, was located on the heavy or light chain or was a conformational determinant, an immunoblotting technique in conjunction with SDS-PAGE was used as described elsewhere (16). Briefly, mAb B3 monoclonal antibody was subjected to SDS-PAGE at a concentration of $1 \mu \mathrm{g} / \mathrm{ml}$ per lane. Each lane was subjected to electrophoretic transfer and rabbit anti- $\lambda$ chain (Sigma Immunochemicals), rabbit anti- $\gamma$ chain (Sigma Immunochemicals), and the anti-Id were added to the separated lanes and binding was detected by a mouse monoclonal anti-rabbit alkaline phosphatase conjugate. To assess whether the binding of the anti-Id to B3 is exclusively directed towards the light chain, the concentration of $\mathrm{mAb} B 3$ was increased to 5 and $10 \mu \mathrm{g} / \mathrm{lml}$ in the lanes, and three concentrations of the anti-Id $(0.5,2$, and $10 \mu \mathrm{g} / \mathrm{ml})$ were used. The intensities of the bands were analyzed by densitometry. Blots were scanned using a Hewlett-Packard Scanjet 2C scanner connected to an Apple MacIntosh IIfx running National Institutes of Health Image software with a gel-plotting macro.

ELISA for detection of B3-Id on immunoglobulins in sera. A capture ELISAs was used to detect the B3-Id in human sera. A 96-well ELISA plate was divided into two. One half was coated with the affinity-purified anti-idiotype (concentration $=1 \mu \mathrm{g} / \mathrm{ml}$ ), while the other half of the plate was coated with the same concentration of the control rabbit immunoglobulin. Human sera (dilution 1 in 300, which gave low binding with the control rabbit immunoglobulin) was applied to both sides of the plate and the binding of IgG and IgM was detected using an alkaline phosphatase goat anti-human IgM or IgG (Fab2) conjugate (Sigma Immunochemicals). The idiotype levels in the sera were calculated by subtracting the binding of the sera to the control immunoglobulin coated wells from the binding of the sera to the antiidiotype-coated wells. The results were then expressed as a percentage of a positive control in duplicate used on all the plates studied. The positive control for the IgG ELISA was the serum of the patient from whom the monoclonal was derived. This control represents the value produced in the Id ELISA and does not represent the percentage of antibodies that bear the idiotype. We used this control to reduce the interassay variation by always comparing the OD readings of the other sera to the OD reading of this sera. The positive control used for the IgM ELISA was a different patient, since the positive control for the IgG idiotype did not have the idiotype on the IgM antibodies present in the serum. Four healthy controls were used on each plate. The upper limit of normal was set as the mean +2 SD of values obtained for the normal control sera.

Preparation of DNA immunosorbent column. Cellulose coupled to ssDNA and dsDNA was purchased from Pharmacia Fine Chemicals; the serum was diluted with $0.1 \mathrm{M}$ Tris-HCL, $\mathrm{pH} 7.4$, containing $0.1 \%$ BSA; $3 \mathrm{M} \mathrm{NaCl}$ was used to elute the column. The specificity and absorption capacity of the column was determined using anti-DNApositive and-negative sera. All serum samples required two absorption cycles to remove all DNA binding antibodies as detected by ELISA. The effluents and eluates were tested separately for anti-Id activity and expressed as a percentage of the original serum sample.

Measurement of serum immunoglobulins, rheumatoid factor, and 
DNA antibodies. Serum IgG and IgM were measured using a previously described capture ELISA (17). Rheumatoid factor was measured by two methods, a direct binding ELISA using horse immunoglobulin and the routine latex fixation test. The DNA antibody levels were measured by ELISA test (Cambridge Life Sciences, Cambridge, United Kingdom; upper limit of normal for this assay is regarded as $100 \mathrm{U} / \mathrm{ml}$ ).

Sequencing of B3 light and heavy variable regions chains. Total RNA was extracted from the cell line via the guanidium thiocyanate/ cesium chloride method (18). $1 \mathrm{mg}$ of total RNA was used to make cDNA using Moloney murine leukemia virus reverse transcriptase (Gibco BRL, Gaithersburg, MD) and random hexamer primers (19). The immunoglobulin variable regions were then amplified by PCR under the following conditions: The $\mathrm{V} \lambda$ region was amplified with a mixture of primers at the 5 ' end (20), which were modified from those described (Hawkins, R., D. Zhu, M. Ovecka, F. K. Stevenson, and G. Winter, manuscript in preparation ). These were used with a mixture of primers for the $\mathrm{J}$ region (20) under the following conditions: $1 \mathrm{~min}$ at $94^{\circ} \mathrm{C}, 1 \mathrm{~min}$ at $65^{\circ} \mathrm{C}$, and $1 \mathrm{~min}$ at $72^{\circ} \mathrm{C}$ for 30 cycles.

The $\mathrm{VH}$ region was amplified using a primer specific for the conserved first eight codons of VH (5'-CAGGTGCAGCTGGTGGAGTCTGG- $\left.3^{\prime}\right)(21)$ and a primer specific for the $C \gamma$ region ( $5^{\prime}$ CAGGGGGAAGACCGATGG3') (22). Amplification was performed under the following conditions: $1 \mathrm{~min}$ at $94^{\circ} \mathrm{C}, 1 \mathrm{~min}$ at $42^{\circ} \mathrm{C}$, and 1 minute at $72^{\circ} \mathrm{C}$ for 30 cycles. The amplified products were electrophoresed through a $1.5 \%$ agarose gel and purified (Geneclean II; Bio 101 Inc., La Jolla, CA). The purified DNA from the $\mathrm{V}$ regions were cloned into a TA cloning vector (Novagen, Madison, WI) and transfected into Escherichia coli strain JM109. Single-stranded DNA was obtained from positive clones using VMSM13 helper phage (Stratagene, La Jolla, CA) and sequenced via the dideoxy chain termination method using the Sequenase kit (U. S. Biochemical Corp., Cleveland, OH). Sequencing was performed on 10 clones derived from two independent PCR products to check for amplification errors. The variable region sequences were analyzed by the PC Gene data base (updated June 1993) to predict the germline gene of origin.

Statistics. Chi-squared analysis or Fisher's exact test was used where appropriate. Kendall's rank correlation was used to assess the significance of the relation between B3-Id and various parameters.

\section{Results}

Characterization of the B3-Id. The specificity of the polyclonal anti-Id-B3 reagent for mAb B3 both in solid phase and fluid phase is shown in Fig 1. Anti-Id-B3 did not react with the control $\mathrm{mAb}$ or pooled IgG. Western blotting demonstrates that the B3-Id is present on the light chain, $\lambda$, of the $\mathrm{mAb}$ (Fig. $2 a$ ). A semiquantitative analysis to determine whether the binding of the anti-idiotype to the monoclonal B3 was specific to the light chain was performed using Western blotting followed by densitometry (Fig. $2 b$ ). The binding of the anti-Id to the heavy chain was not significantly above that found with the normal rabbit serum at the highest concentration of B3 and anti-Id. Therefore, Id is almost certainly confined to the light chain. By inhibition ELISA, dsDNA but not ssDNA or RNA blocked the binding of $\mathrm{B} 3$ to the anti-Id demonstrating that the Id is at, or close to, the binding site of the mAb B3 (Fig. 3). Therefore, the light chain is involved in the binding of $\mathrm{B} 3$ to dsDNA.

Expression of the B3-Id in sera. Based on tests of 28 healthy controls, the upper limit of normal of the idiotype expression on IgG was set at $34 \%$ (percent of the positive control) and the upper limit of normal of the idiotype expression on IgM was set at $22 \%$. Fig. 4 shows the level of expression of the idiotype on IgG and IgM antibodies in the six different organ systems stud-
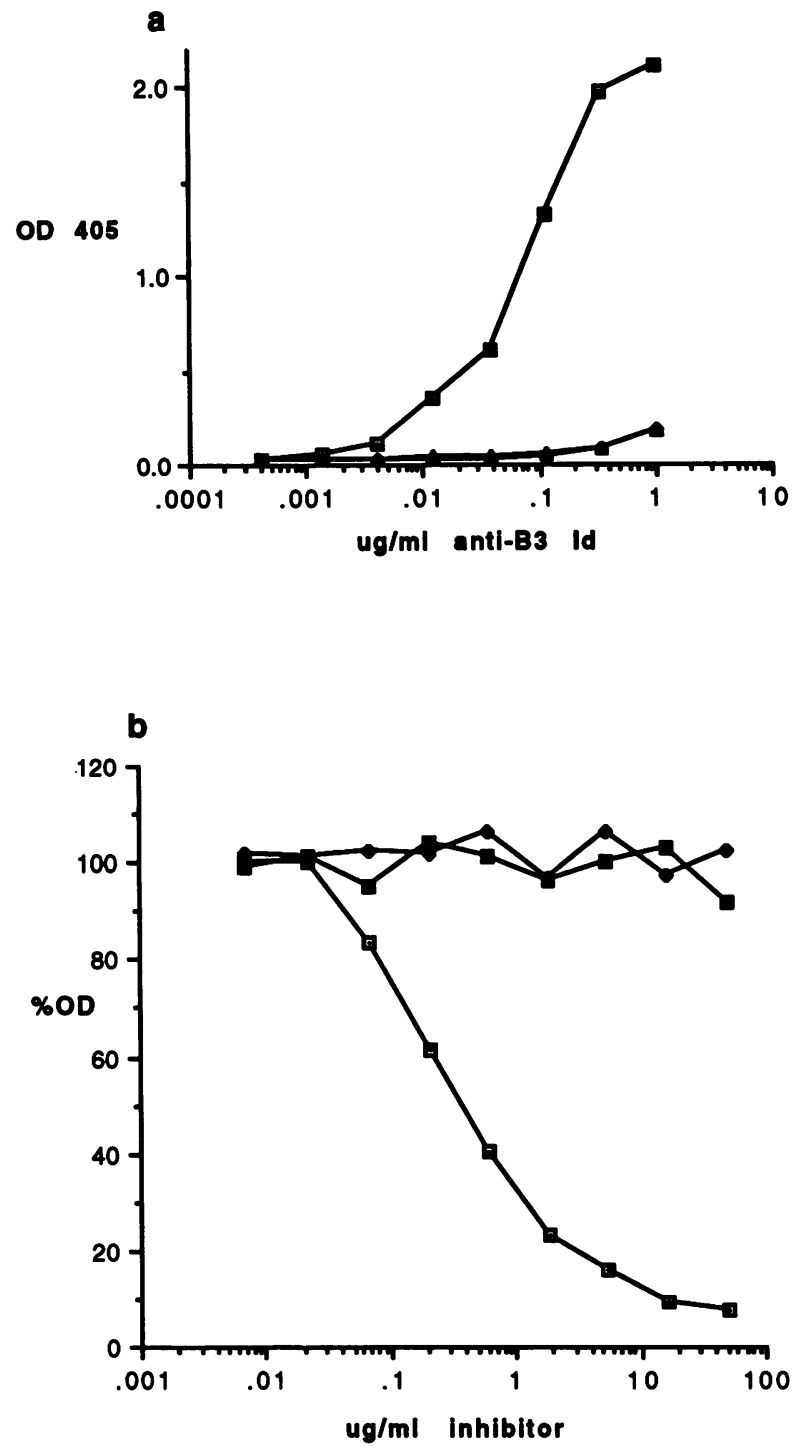

Figure 1. (a) Binding of anti-Id-B3 to solid-phase B3, pooled human IgG, and RSP-1, a human IgG monoclonal antibody of unknown specificity, in an ELISA. Results are expressed as OD readings. $-\square$ ,$- \mathrm{B} 3 ;-\longrightarrow, \mathrm{RSP}-1 ;-\wedge-$, pooled IgG. $(b)$ Inhibition of the binding of anti-Id-B3 to B3 in solid phase by fluid-phase B3, pooled IgG, and RSP-1. \%OD, percent binding of anti-Id-B3 to B3 compared to the binding without inhibiting antigen. $-\longrightarrow, \mathrm{B} 3$; $-\longrightarrow$, pooled IgG; $\longrightarrow$-, RSP-1.

ied in the SLE patients and in the other groups (Table I provides a summary). When present, the idiotype is expressed more frequently on IgG antibodies compared to IgM ( $P$ $<0.001)$ in SLE patients, whereas in the rheumatoid arthritis population, it is expressed more frequently on the IgM population $(P<0.05)$. Within the SLE cohort, the B3-Id was present more frequently in those patients with active $(M / S)$ disease $(42 \%)$ compared to patients with the other manifestations ( $P$ $<0.001)$. The serum of the 15 SLE patients that had significantly raised levels of B3-Id on IgG antibodies associated with active arthritis were also tested when their disease was inactive. Four of these patients' sera were also examined on a number of occasions (see serial studies below). Out of the other 11 patients that were examined on one other occasion, none had elevated B3-Id levels when their arthritis was inactive (data not 
shown). In addition, 10 patients with active arthritis that were B3-Id negative were also tested when their disease was inactive. In all cases, the sera was negative for the idiotype.

Out of the 35 patients with $\mathrm{M} / \mathrm{S}$ disease, only three had myositis, all of whom were negative for the idiotype, the other patients had joint disease. There was no difference in the clinical expression of the joint disease between those patients who were idiotype-positive and idiotype-negative. 79 of the 101 SLE patients, in comparison with 16 out of 20 B3-Id positive patients, had anti-DNA antibodies, indicating that the antiidiotype did not select out patients whose serum bound DNA. The one rheumatoid arthritis patient that had the B3-Id on the IgG population had detectable anti-DNA antibodies by ELISA (though not by Crithidia immunofluorescence). One out of the six SLE patients with B3-Id on the IgM population had an erosive arthritis as well. The level of expression of B3-Id on IgM was higher in the rheumatoid arthritis patients compared to the other groups.

Serial studies. Serial samples were taken from six idiotype positive patients to analyze the variation of the idiotype level over time. In the four patients with active arthritis, the level of B3 Id followed the musculoskeletal disease activity more closely than the overall disease activity without the musculoskeletal component (Kendall rank correlation $P<0.05$, Fig. $5)$. The correlation between the B3 Id level and arthritis activity did not hold in the two patients who had raised idiotype levels in the serum taken at the time they had active skin disease (also shown in Fig. 5). The variation in idiotype levels in these two patients did not correlate with the variation in the BILAG score of any of the individual systems nor of the global score. When the disease was inactive, the idiotype was not found on IgM antibodies in any of the patients studied. The correlation of B3-Id with DNA binding seems to be less obvious.

The correlation between the idiotype level on IgG antibodies and the total IgG level was not significant (Fig. $6 a$ ). Fig. $6 b$ illustrates the lack of association between rheumatoid factor levels and the expression of the B3-Id on IgM antibodies in rheumatoid patients.

Serum absorption studies. After absorption of four individual SLE serum samples that were positive for DNA binding on a DNA-cellulose column, anti-DNA activity was unmeasurable. In the four patients studied between 46 and $64 \%$ of the B3-Id was present in the DNA binding fraction eluted from the column (individual data not shown). Thus, a sizable fraction of the DNA antibodies in the serum of these patients had the B3-Id.

Sequence analysis. The nucleotide sequence of the $\mathrm{V} \lambda$ region of the IgG anti-dsDNA antibody B3 (Fig. $7 a$ ) indicates that it has 90\% homology with the germline gene $V \lambda 2.1$ (23), and $85 \%$ homology at the amino acid level Fig. $7 \mathrm{~b}$. There is a total of 14 replacement mutations, 6 of which are in CDR3. The replacement to silent ratio in CDR3 of the light chain is 7 , implying a possible antigen-driven mechanism. The $J \lambda$ region is unmutated $\mathrm{J} \lambda 2$. A sequence comparison between $\mathrm{B} 3$ and PV11, an SLE-derived, IgM non-DNA binding $\mathrm{mAb}$ that carries the 8.12 Id (24) revealed a 96\% homology at the nucleotide level and $89 \%$ homology at the amino acid level. There were four changes to positively charged amino acids (three arginines), known to be involved in DNA binding, in the CDRs of B3 $\lambda$ chain compared to none in the $\lambda$ chain of PV11.

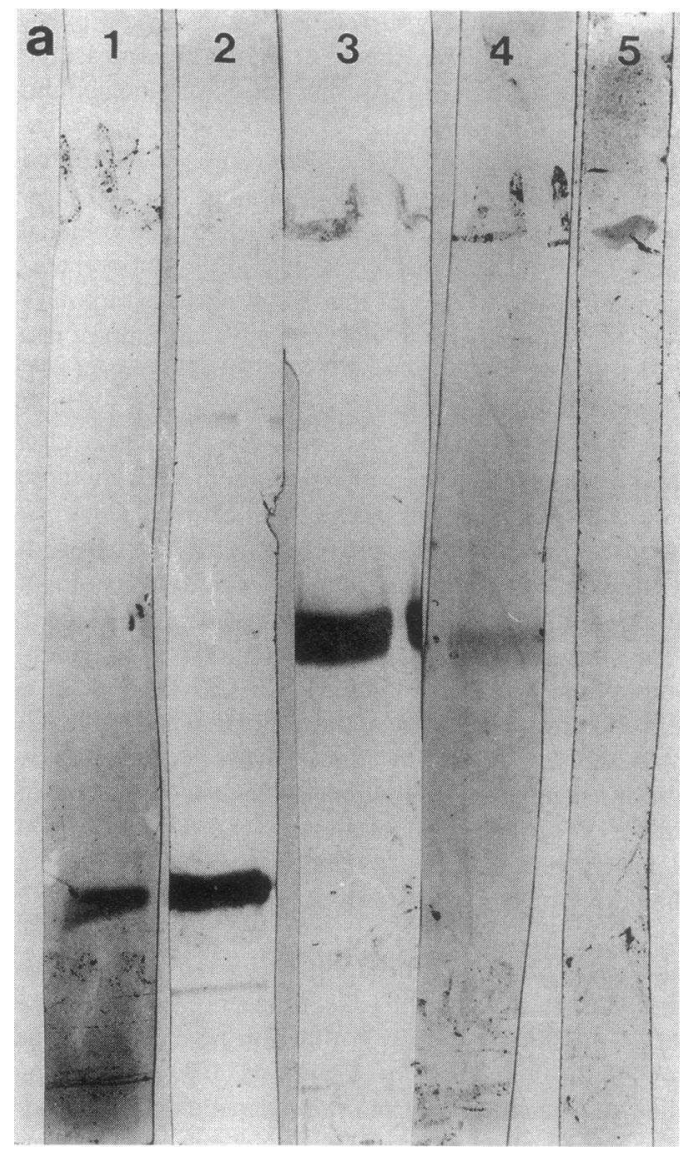

Figure 2. (a) Western blots of reduced B3 ( $1 \mu \mathrm{g} /$ lane) run on a $10 \%$ polyacrilamide gel, stained with anti-Id-B3 (lane 1), rabbit antihuman $\lambda$ (lane 2), rabbit anti-human $\gamma$ (lane 3), normal rabbit serum (lane 4), and PBS (lane 5). (b) Analysis of Western blots by densitometry using higher concentrations of $\mathrm{mAb}$ and anti-Id than in $a$. Lanes 1-6 represent blots with $5 \mu \mathrm{g} /$ lane of mAb B3; lanes 7-12 have $10 \mu \mathrm{g} /$ lane of B3. Lanes $1-3$ and 7-9 were incubated with increasing concentrations of anti-Id $(0.5 \mu \mathrm{g} / \mathrm{ml}, 2 \mu \mathrm{g} / \mathrm{ml}$, and $10 \mu \mathrm{g} /$ $\mathrm{ml}$ ); lanes 4 and 10 were incubated with $2 \mu \mathrm{g} / \mathrm{ml}$ of normal rabbit serum; and lanes 5 and 11 , and 6 and 12 were incubated with anti- $\lambda$ and anti- $\gamma$ chain, respectively. There is no significant binding of the anti-Id to the heavy chain above that seen with the normal rabbit serum.

The nucleotide sequence of the heavy chain of B3 (Fig. $8 a$ ) reveals that it has $93.5 \%$ homology with the germline gene segment VH26 (25) and 89\% at the amino acid level. The D region could be aligned to the $\mathrm{D}$ gene segment $\mathrm{DN1}$. The CDR3 region contains seven amino acids including one asparagine residue. There are no charged residues present in the CDR3 region. There is a replacement mutation at position 54 in CDR2 from serine to arginine resulting in a gain in positive charge. Lysine is replaced by glutamine at position 65 where a positive charge is lost. There are nine other replacement mutations within the $\mathrm{VH}$ region none of which lead to any change in charge. The framework 4 region consists of a JH4 gene segment with one mutation from serine to tyrosine.

\section{Discussion}

The B3-Id is the first idiotype to be defined by a rabbit polyclonal anti-Id raised by a human monoclonal IgG anti-dsDNA 


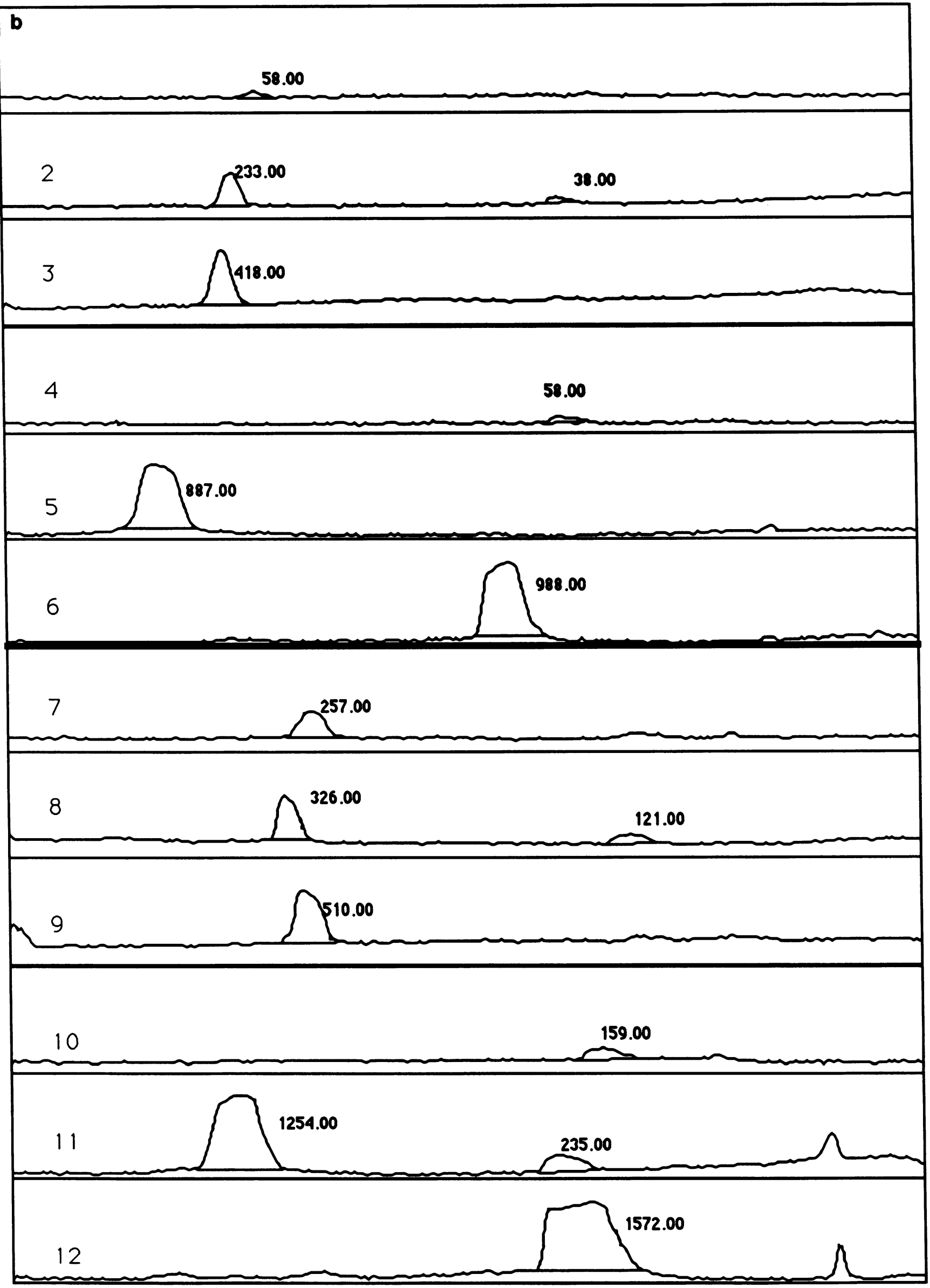




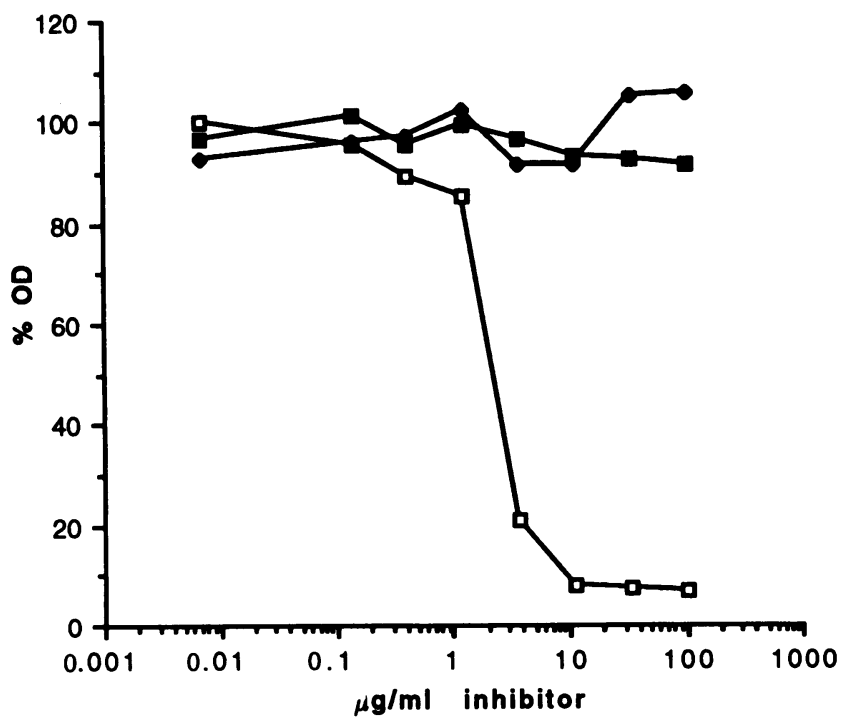

Figure 3. Inhibition of the binding of $\mathrm{B} 3$ to anti-Id-B3 in solid phase by dsDNA, ssDNA, and RNA. ——-, dsDNA; - - , ssDNA; $\rightarrow-$ RNA.

antibody. The idiotype, when detected, appears to be present predominantly on the IgG population in SLE sera but also intriguingly on the IgM population of sera from some rheumatoid arthritis patients. This did not merely reflect an overall increase in IgG in SLE since there was no correlation between the B3-Id level and total IgG levels. Thus, the increase in idiotype levels is unlikely to be simply caused by hypergammaglobulinaemia secondary to polyclonal activation. In patients with primary Sjögren's syndrome, the B3-Id was not detectable either on IgM or IgG antibodies. In contrast, other idiotypes identified on anti-DNA antibodies are frequently found in other autoimmune rheumatic diseases, particularly Sjögren's syndrome. In a collaborative study of 19 different idiotypes, nine were present in $>30 \%$ of Sjögren's syndrome, and none of the idiotypes were disease specific (2).

The increased expression of this idiotype in SLE patients is largely confined to lupus patients who have active arthritis, though only a proportion of these were positive. Moreover, the level of B3-Id showed a closer correlation to arthritis activity than to overall disease activity in four patients studied serially. Since SLE often involves several organs at the same time, though to variable degrees, previous attempts at linking the levels of idiotypes to disease using global indices may have failed to detect underlying disease fluctuations in individual
\% Id B3G a

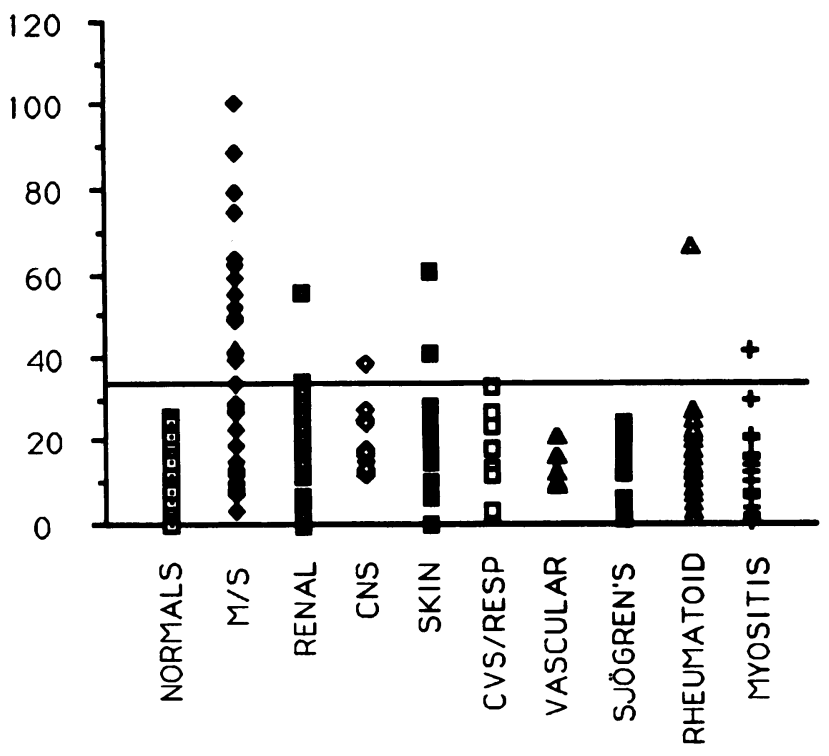

\% Id B3M b

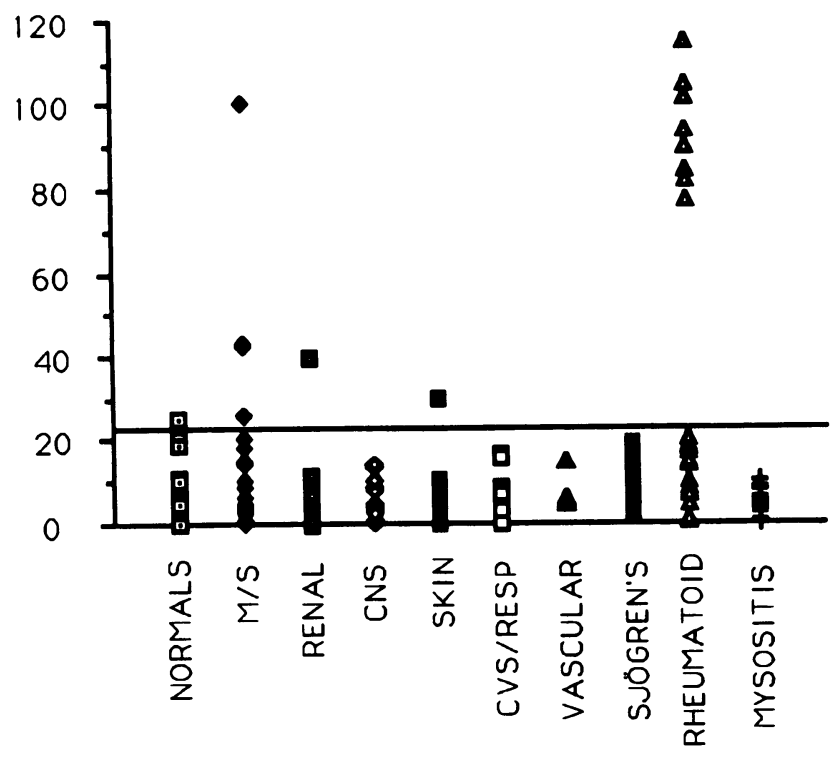

Figure 4. B3-Idiotype levels on $\operatorname{IgG}(a)$ and $\operatorname{IgM}(b)$ in the sera of patients with SLE classified according to organ involvement, normal controls, and other autoimmune rheumatic diseases Data are expressed as a percentage of a positive control. Horizontal line represents the mean +2 SD from the mean.

Table I. Expression of the B3-Id on IgG and IgM Antibodies in Serum from Patients with SLE, Normal Controls, and Other Autoimmune Rheumatic Diseases

\begin{tabular}{lrrrrrrrrrrr}
\hline & M/S & Renal & CNS & Skin & CVS/Resp & Vasc & SLE & RA & Sjögren's & Myositis & Normals \\
\hline Total & 35 & 24 & 14 & 16 & 8 & 4 & 101 & 27 & 20 & 17 \\
B3G & 15 & 2 & 1 & 2 & 0 & 0 & 20 & 1 & 0 & 1 \\
B3M & 4 & 1 & 0 & 1 & 0 & 0 & 6 & 8 & 0 & 0 \\
\end{tabular}

Values represent numbers of patients in each group. SLE patients divided according to BILAG index (see Methods). The column headed "Myositis" includes only patients with idiopathic myositis not related to SLE. 

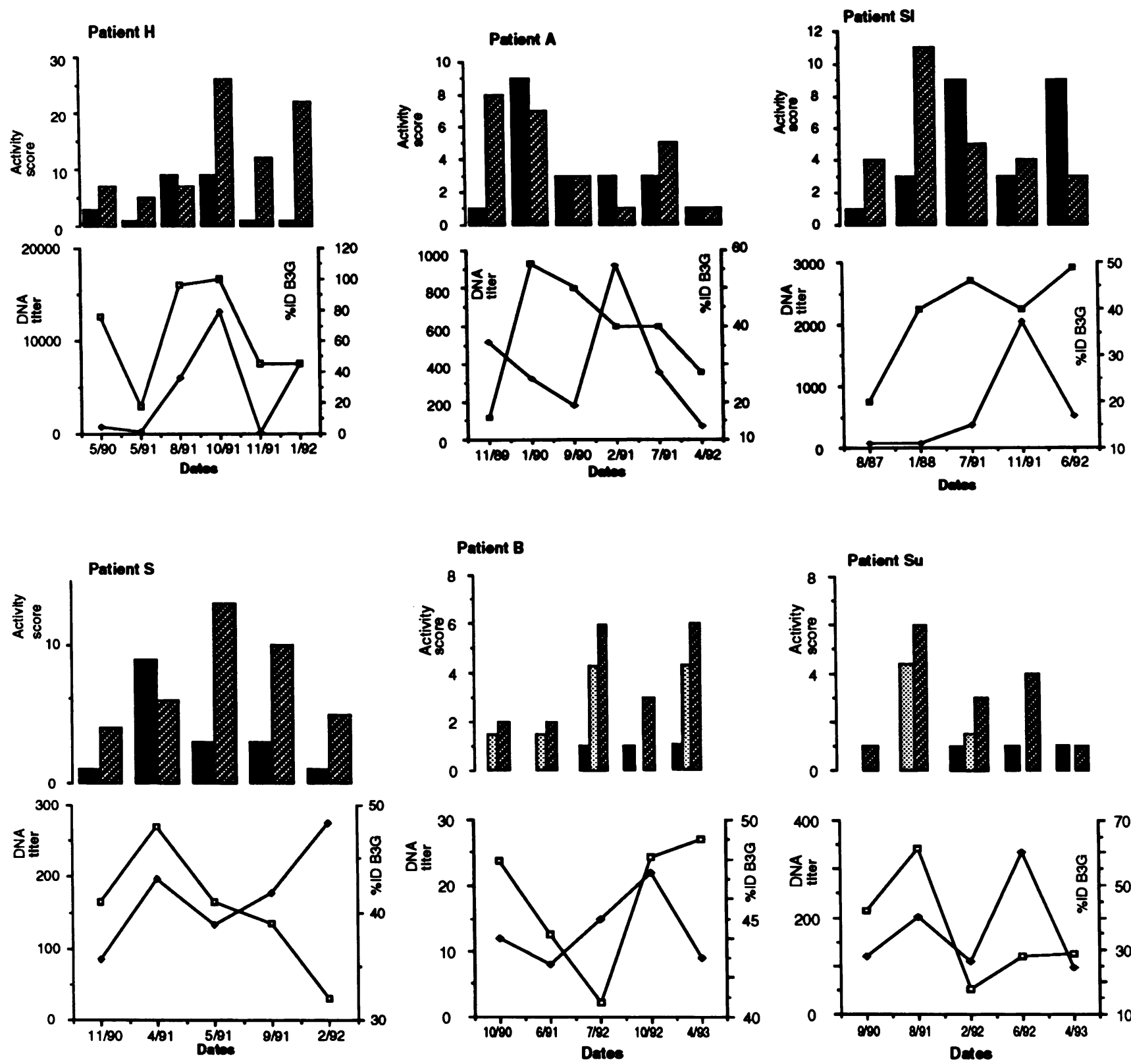

Figure 5. Serial bleeds from six B3-Id-positive SLE patients comparing levels of B3-Id (\%ID B3G) to M/S disease activity and overall activity without the musculoskeletal component. (Disease activity was assessed by the BILAG system, which can yield a global score; see Methods). In the four patients who had active musculoskeletal disease, the correlation between the musculoskeletal disease activity and the idiotype level was significant (Patient SI, $P=0.004$; Patient A, $P=0.001$; Patient $\mathrm{H}, P=0.004$; Patient $\mathrm{S}, P=0.05$; Kendall rank correlation). There was no correlation between the B3-Id level and the overall disease activity without the $\mathrm{M} / \mathrm{S}$ activity. The other two patients were the only patients that had active skin disease (Patient B, Patient $\mathrm{Su}$ ) when the B3-Id level was raised, but there was no correlation with disease activity. (Skin disease activity is shown separately for these two patients.) Also illustrated are the serial levels of DNA binding. $-\bullet$, , DNA; $\longrightarrow \square-, \%$ ID B3G; $\bullet$, M/S disease activity; , Other disease activity; $\square$, Skin disease activity.

systems. Using the BILAG index, which was designed to distinguish individual organ/system involvement and incorporates a rate of change element, allows the fine dissection of disease activity that we described.

A further source of obfuscation may arise from the failure to distinguish which individual immunoglobulin class the idiotypes are present on. Of the separate analysis of IgG and IgM antibodies that have been reported in the literature, the $16 / 6$ idiotype has been found to be expressed on both IgG and IgM antibodies in tissue lesions (26). The PR4-Id derived from a patient with leprosy has been found to be expressed on $\mathrm{IgG}$, IgM, and IgA antibodies in lupus patients, but no correlation was found between the IgG, IgA, and IgM PR4-Id levels and a variety of parameters in lupus patients including disease activity (27). The 3I idiotype has been found on both IgG and IgM antibodies; anti-DNA binding was not associated with the IgM class on myeloma proteins but was on SLE derived IgG and IgM monoclonal antibodies derived from EBV transformed 

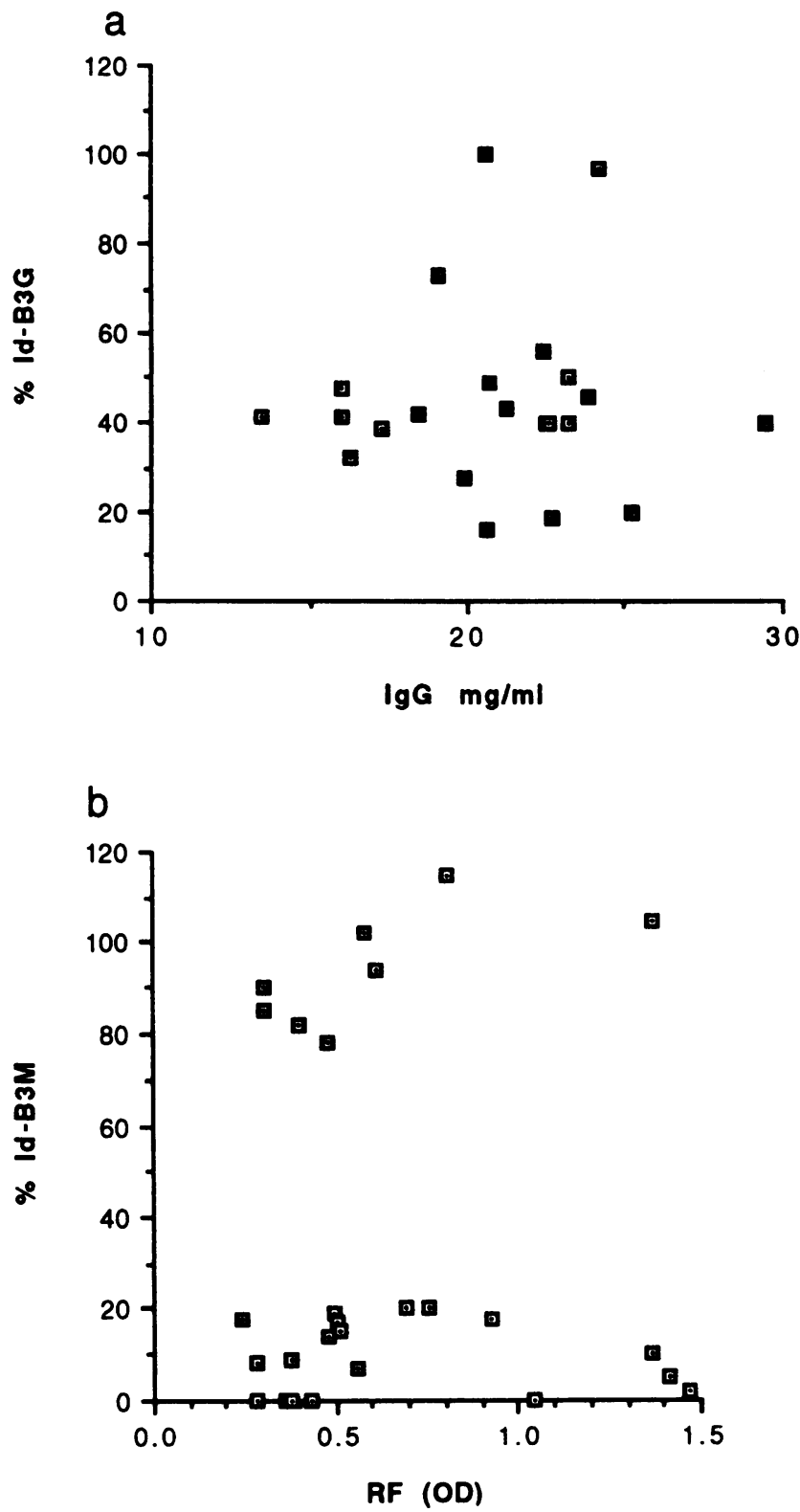

Figure 6. (a) Correlation between the IgG concentration and the IgG B3-Id levels found in some of the SLE patients. ( $b$ ) Correlation between rheumatoid factor levels and B3-Id levels on IgM antibodies in rheumatoid arthritis patients.

lymphocytes (3). Switching of an idiotype from one immunoglobulin class to another may be linked to the development of more pathogenic antibodies. The B3-Id does not appear to be switch from IgM antibodies to IgG antibodies since it is rarely present on IgM antibodies in normal individuals or SLE patients, rather it may be associated with IgG antibodies in the context of active SLE. An idiotype derived from the purified serum of an SLE patient known as F4 was also found almost exclusively on IgG antibodies (28). This idiotype is located on the heavy chain but is associated with 3I idiotype expression found on the $\kappa$ chain. The B3-Id is however also present on IgM antibodies in rheumatoid arthritis (see later discussion).
The nucleotide sequence of the $\lambda$ chain of $\mathrm{B} 3$ shows that it is $90 \%$ homologous at the nucleotide level with the V $\lambda$ II gene family used to encode the pathogenic 8.12 idiotype (24) and indeed B3 is 8.12 Id positive (Diamond, B., unpublished observation). This idiotype has been found in immune complexes found in the kidney lesions of SLE patients. The 8.12 Id differs from B3-Id in its distribution and because the 8.12 Id is not located near the DNA binding site. The highest homology (96\%) out of a bank of eight 8.12 Id-positive and two 8.12 Id-negative antibodies, all of which use the V $\lambda$ II gene family, is seen with the monoclonal antibody PV11. This monoclonal IgM, 8.12-positive antibody does not bind to DNA. Although the heavy chain of B3 probably contributes to DNA reactivity, the anti-Id, which binds to the light chain, does inhibit DNA binding. Comparing the amino acid sequence of the light chain of B3 and PV11, there are four mutations to positively charged amino acids (three arginines) in B3, a couplet of arginines are present in CDR1. These amino acids, such as arginine, have been shown to be important in conferring DNA specificity in murine anti-DNA antibodies (29). The data presented here suggests that these observations might apply to the human $\lambda$ chain as well. PV11 has been found to be negative for B3-Id (Ehrenstein, M., and B. Diamond, unpublished observation), and a comparison between the VL sequences of the two antibodies might indicate possible sites where the idiotype or part of the idiotype is situated. However, to localize the position of the idiotype, further epitope mapping and site-directed mutagenesis studies need to be performed.

The CDR 1 of the $\kappa$ chain may be important in DNA binding of 3I idiotype-positive antibodies (30). Indeed, Diamond and colleagues conclude that DNA binding by 3I-positive antibodies may be more a function of the light chain than the heavy chain. There are two important differences, however, between the 3I-positive antibodies and B3. Firstly, the 3I-positive $\kappa$ chains that bind DNA are more negatively charged than their germline counterparts and secondly, the heavy chains of the 3I-positive antibodies show evidence for antigen selection, whereas the heavy chain of B3 shows a high homology to VH26 without any evidence for antigen selection.

The germline gene VH-26, from which the nucleotide sequence of B3 is likely to be derived, encodes the $16 / 6$ Id serum levels of which correlate with disease activity in patients with SLE (2). The replacement mutation of serine to arginine at position 54 is the only amino acid change likely to enhance DNA binding. In addition, the asparagine residue in CDR3, probably coded for by $\mathrm{N}$ addition, could also contribute to DNA binding. Arginine can form two hydrogen bonds with guanine in double-stranded DNA, and asparagine can hydrogen bond to adenine (31).

Since IgG antibodies are more frequently, though not exclusively, associated with a secondary immune response where antigen stimulation plays an important part, evidence of antigen selection, that is increased replacement to silent mutations in the CDRs of the antibodies, would be expected to occur. Interestingly, only the replacement to silent mutation ratio in the CDR 3 of $B 3 \lambda$ chain, which is 7 , indicates a possible antigen-driven mechanism (32). Though the germline gene from this patient has not been determined, and thus these figures may not be entirely accurate, the link with the 8.12 idiotype does lend support to this conclusion. The somatic mutation 
b
F R 1
CDR 1
F R 2
CDR2

V 22.1 QSALTQPASVSGTPGQSI TISC TGTSSDVGSYNLVS WYQQHPGKAPKLMIY EGSKRPS

B3

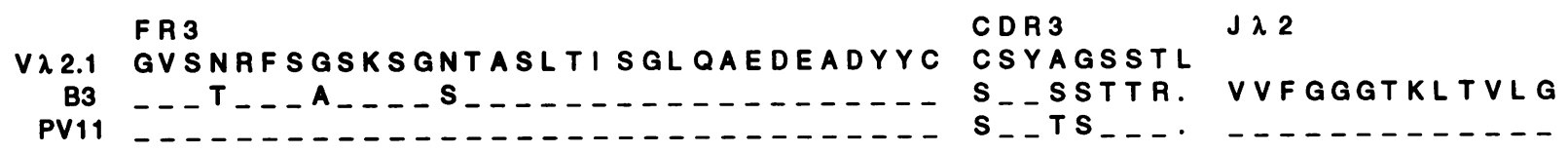

Figure 7. The nucleotide $(a)$ and amino acid $(b)$ sequences of B3 aligned to V 2.1 and PV11, an 8.12 positive antibody. Dashes indicate identity to $\mathrm{V} \lambda 2.1$ and dots are inserted to facilitate alignment. Germline sequence of $\mathrm{J} \lambda 2$ is reproduced from reference 34 . The sequence data in this figure has been assigned an accession number from Gen Bank: L22483.

may not only yield anti-DNA antibodies, but also specificities or particular idiotypes associated with arthritis. Moreover, the eliciting antigen may not be DNA, and amino acid substitution may occur to provide increased binding to an unidentified antigen. The DNA absorption studies and other data indicate that the idiotype is present on antibodies that do not bind DNA, as well as on antibodies that do bind DNA. Interestingly, IgM rheumatoid factors have been found to bind DNA/histone complexes (33). This provides a link between the elevation of the B3Id and the joint disease seen in SLE and rheumatoid arthritis. This link is potentially strengthened because the monoclonal B3 binds DNA/histone complexes, a feature not common to all DNA binding antibodies (8). An alternative possibility to explain the presence of B3-Id on IgM antibodies in rheumatoid arthritis, although we do not favor it, is that the anti-idiotype is recognizing a slightly different epitope from that "seen" in patients with SLE.

Tissue studies have found the deposition of idiotypes such as 16/6, GN-2, and 9G4 in pathological lesions in kidneys and skin $(26,4,5)$, but it is rarely practical to obtain synovial biopsies of patients with SLE as there is usually little clinical indication. The B3-Id was not found in 10 renal biopsies of SLE patients (data not shown).

In summary, we have described the first anti-DNA antiidiotype derived from a human monoclonal anti-dsDNA antibody. This idiotype, when present on IgG antibodies, is associated with a proportion of SLE patients with active arthritis. Moreover, it appears that this idiotype is expressed on IgM antibodies in some patients with rheumatoid arthritis. Sequence analysis implies that positively charged amino acids on the $\lambda$ chain may contribute to DNA binding.

\section{Acknowledgments}

We would like to thank Mr. Steven Cheung for assistance with the immunoglobulin assay and Mr. Paul Levy for assistance with the animals used to generate the anti-idiotypes.

We gratefully acknowledge the support of the Arthritis and Rheumatism Council of Great Britain. 
a

\begin{tabular}{|c|c|c|c|c|c|c|c|c|c|c|c|c|c|c|c|c|c|c|c|}
\hline VH26 & $\begin{array}{l}F \text { P 1 } \\
\text { GA G }\end{array}$ & GT G & CAG & C T G & T T G & GA G & TCT & GGG & GGA & $\mathbf{G G C}$ & T T G & GT A & CAG & CCT & GGG & $\mathbf{G G G}$ & T C C & C T G & $A G A$ \\
\hline B3 & --- & --- & --- & -- & $G_{--}$ & --- & -- & -- & --- & -- & --- & $\cdots$ & --- & -- & -- & --- & $\cdots$ & $--C$ & -- \\
\hline 6 & СТ C & TCC & $\mathbf{T} \mathbf{G T}$ & GCA & CC & $T C T$ & GGA & T T C & $A C C$ & T T T & A GC & $\begin{array}{l}\text { CDR1 } \\
\text { A GC }\end{array}$ & TAT & GC C & A T G & A GC & $\begin{array}{l}\text { F R2 } \\
\text { T GG }\end{array}$ & G T C & CGC \\
\hline B3 & --- & --- & $-\cdots$ & --- & $\mathbf{A} \mathbf{G}_{-}$ & --- & --- & -- & --- & -- & $-T_{-}$ & $-C_{-}$ & -- & --- & -- & --- & --- & --- & $\ldots$ \\
\hline . . i<6 & CAG & GCT & CCA & GGG & $A \mathbf{A G}$ & $\mathbf{G G G}$ & $\mathbf{C} \mathbf{T} \mathbf{G}$ & $\mathbf{G A G}$ & $\mathbf{T} \mathbf{G G}$ & GT C & TCA & $\begin{array}{l}\text { CDR2 } \\
\text { GCT }\end{array}$ & ATT & A G T & GG T & A GT & GG T & GGT & A GC \\
\hline B3 & --- & --- & --- & --- & --- & --- & --- & --- & --- & --- & --- & $A_{--}$ & --- & --- & $\cdots$ & $C_{-}$ & --- & $A_{--}$ & --- \\
\hline $\begin{array}{c}\text { VH2B } \\
\text { B3 }\end{array}$ & ACA & TAC & TAC & GCA & GAC & TCC & GT G & $A A G$ & GGC & $\begin{array}{l}\text { FR3 } \\
\text { CGG }\end{array}$ & T T C & ICC & A T C & T C C & A GA & GAC & A A T & T C C & $\mathbf{G}$ \\
\hline & & --- & --- & --1 & --- & --- & --- & -. & $\cdots$ & --- & --- & $\cdots$ & c- & --- & --- & $\cdots$ & --- & $\cdots$ & --- \\
\hline VH26 & AAC & ACG & C T G & TAT & CT G & CAA & ATG & $A A C$ & ACG & CT G & AGA & GCC & GAG & GAC & $A C G$ & GCC & GTA & TAT & TAC \\
\hline & $-\boldsymbol{a}_{-}$ & $--c$ & --- & --- & $--c$ & $--G$ & --- & --- & - GC & --- & --- & --- & --- & --- & --- & --- & $--C$ & --- & --- \\
\hline$B$ & T GT & $\mathbf{G C G}$ & AAA & CDR3 & & & & & & & $\mathrm{JH}_{4}$ & & & & & & & & \\
\hline Bs & & & & ССТ & AAT & GT G & GOC & AGT & GGC & T GG & TCC & T T T & GAC & TCC & T GG & GGC & CAG & GGA & $A C C$ \\
\hline & & & & & & & & & & & & & & & & & & & \\
\hline
\end{tabular}

b

CDR 1 F R 2

C D R 2

VH26 EVQLLESGGGLVQPGGSLRLSCAASGFTFS SYAMS WVRQAPGKGLEWVS AISGSGG
B3

F R 3

VH26 STYYADSVKG RFTI SRDNSKNTLYLQMNSLRAEDTAVYYCAK

B3 _....... Q $\ldots \ldots \ldots$.

CDR $3 \quad J H_{4}$

B3 PNVGSGWSFDSGQGTLVTVS

Figure 8 . The heavy chain nucleotide $(a)$ and amino acid $(b)$ sequence of the variable region of B3 aligned to VH-26.

\section{References}

1. Watts, R., and D. A. Isenberg. 1990. DNA antibody idiotypes: an analysis of their clinical connections and origins. Int. Rev. Immunol. 5:279-293.

2. Isenberg, D. A., W. Williams, J. Axford, R. Bakimer, D. Bell, T. CasasecaGrayson, B. Diamond, F. Ebling, B. Hahn, and G. Harkiss, et al. 1990. Comparison of DNA antibody idiotypes in human sera. An international collaborative study of 19 idiotypes from 11 different laboratories. J. Autoimmun. 3:393-414.

3. Manheimer-Lory, A. J., A. Davidson, D. Watkins, N. R. Hannigan, and B. A. Diamond. 1991. Generation and analysis of clonal IgM and IgG producing human B cell lines expressing an anti-DNA associated idiotype. J. Clin. Invest. 87:1519-1525.

4. Kalunian, K., N. Panosian-Sahakian, F. Ebling, A. Cohen, J. Louie, J. Kaine, and B. Hahn. 1989. Idiotypic characteristics of immunoglobulins associated with systemic lupus erythematosus. Arthritis Rheum. 32:513-522.

5. Isenberg, D. A., M. Spellerberg, W. Williams, M. Griffiths, and F. Stevenson. 1993. Identification of a role for the 9G4 idiotype in systemic lupus erythematosus. Br. J. Rheum. 32:876-882.

6. Symmonds, D. P. M., J. S. Coppock, P. A. Bacon, B. Bresnihan, D. A. Isenberg, P. J. Maddison, N. McHugh, M. L. Snaith, and A. A. Zoma. 1988. Development of a computerised index of clinical disease activity in systemic lupus erythematosus. Q. J. Med. 69:927-937.

7. Okamura, M., Y. Kanayama, K. Amastu, N. Negoro, S. Kohda, T. Takeda, and T. Inoue. 1993. Significance of enzyme linked immunosorbent assay (ELISA) for antibodies to double stranded and single stranded DNA in patients with lupus nephritis: correlation with severity of renal histology. Ann. Rheum. Dis. 52:14-20.

8. Ehrenstein, M. R., C. M. Longhurst, and D. A. Isenberg. Production and analysis of IgG monoclonal anti-DNA antibodies from SLE patients. 1993. Clin. Exp. Immunol. 92:39-45.

9. Tan, E. M., A. S. Cohen, J. F. Fries, A. T. Masi, D. J. McShane, N. F. Rothfield, J. G. Schaller, N. Talal, and R. J. Winchester. 1982. The 1982 revised criteria for classification of systemic lupus erythematosus. Arthritis. Rheum. 25:1271-1272.

10. Hay, E. M., P. A. Bacon, C. Gordon, D. A. Isenberg, P. Maddison, M. L. Snaith, D. P. M. Symmons, N. Viner, and A. Zoma. 1993. The BILAG index: a reliable and valid instrument for measuring clinical disease activity in systemic lupus erythematosus. $Q$. J. Med. 86:447-458.

11. Gladman, D. D., C. H. Godsmith, M. B. Urowitz, P. Bacon, C. Bombardier, D. A. Isenberg, K. Kalunian, M. H. Liang, and P. Maddison, et al. 1992. Crosscultural validation and reliability of 3 disease activity indices in systemic lupus erythematosus. J. Rheum. 19:608-611.

12. Arnett, F. C., S. M. Edworthy, D. A. Bloch, D. J. McShane, J. F. Fries, N. S. Cooper, L. A. Healey, S. R. Kaplan, M. H. Liang, and H. S. Luthra, et al. 1988. The American Rheumatism Association 1987 revised criteria for the classification of rheumatoid arthritis. Arthritis Rheum. 31:315-324.

13. Isenberg, D. A., D. Rowe, A. Tookman, A. Hopp, M. Griffiths, E. Paice, J. Stewart, and P. C. L. Beverley. 1984. An immunohistochemical study of secondary Sjögren's syndrome. Ann. Rheum. Dis. 43:470-476.

14. Bohan, A., and J. B. Peter. 1975. Polymyositis and dermatomyositis. $N$. Engl. J. Med. 292:344-347. 
15. Watts, R. A., C. T. Ravirajan, N. A. Staines, and D. A. Isenberg. 1990. A human fetal monoclonal DNA-binding antibody shares idiotypes with fetal and adult murine monoclonal DNA binding antibodies. Immunology. 69:348-354.

16. Williams, W., A. Zumla, R. Behrens, M. Locniskar, A. Voller, K. P. W. J. McAdam, and D. A. Isenberg. 1988. Studies of a common idiotype PR4 in autoimmune rheumatic disease. Arthritis Rheum. 31:1097-1010.

17. Watts, R. A., W. Williams, S. LePage, A. Norden, A. Soltys, G. Swana, I. Addison, F. C. Hay, and D. A. Isenberg. 1989. Analysis of autoantibody reactivity and common idiotype PR4 expression of myeloma proteins. J. Autoimmun. 2:689-700.

18. Chirgwin, J. M., A. E. Przbyla, R. J. H. MacDonald, and W. J. Rutter 1979. Isolation of biologically active ribonucleic acid from sources enriched in ribonuclease. Biochemistry. 18:5294-99.

19. Kawasaki, E. 1990. Amplification of RNA. In PCR Protocols: A Guide to Methods and Applications. M. A. Innis, J. Sninsky, and T. J. White, editors Academic Press, California p21-27.

20. Marks, J. D., M. Tristem, A. Karpas, and G. Winter. 1991. Oligonucleotide primers for polymerase chain reaction amplification of the human immunoglobulin variable genes and design of family-specific oligonucleotide probes. Eur. J. Immunol. 21:985-991.

21. Watts, R., J. Hilson, J. Oppilger, L. MacKenzie, P. M. Lydyard, C. G. Mackworth Young, C. Brown, N. A. Staines, and D. A. Isenberg. 1992. Sequence analysis and idiotypic relationships of BEG-2 a human fetal antibody reactive with DNA. Lupus. 1:9-17.

22. Stevenson, F. K., C. Longhurst, C. J. Chapman, M. R. Ehrenstein, M. B. Spellerberg, T. J. Hamblin, C. T. Ravirajan, D. Latchman, and D. A. Isenberg Utilization of the VH4-21 gene segment by anti-DNA antibodies from patients with SLE. 1993. J. Autoimmun. 6:809-826.

23. Brockly, F., D. Alexandre, P. Chuchana, S. Huck, G. LeFranc, and M. P. LeFranc. 1989. First nucleotide sequence of a human immunoglobulin variable lambda gene belonging to subgroup II. Nucleic Acids Res. 17:3976.

24. Paul, E., A. A. Iliev, A. Livneh, and B. Diamond. 1992. The anti-DNA-as- sociated idiotype 8.12 is encoded by the VI2 gene family and maps to the vicinity of L chain CDR1. J. Immunol. 149:3588-3595.

25. Chen, P. P., M. F. Liu, S. Sinha, and D. Carson. 1988. A 16/6 idiotype positive anti-DNA antibody is encoded by a conserved VH gene with no somatic mutation. Arthritis Rheum. 11:1429-1432.

26. Isenberg, D. A., and C. Collins. 1985. Detection of cross-reactive antiDNA antibody idiotypes on renal tissue bound immunoglobulins from lupus patients. J. Clin. Invest. 76:287-289.

27. Pilcher, C., W. Williams, and D. A. Isenberg. 1991. Assessment of common idiotype PR4-Id in serial bleeds from lupus patients. Autoimmunity. 9:7-12.

28. Davidson, A., A. Smith, J. Katz, J. L. Preud'homme, A. Solomon, and B. Diamond. 1989. A cross-reactive idiotype on anti-DNA antibodies defines an $\mathrm{H}$ chain determinant present almost exclusively on IgG antibodies. J. Immunol. 143:174-180.

29. Radic, M. Z., J. Mackle, J. Erikson, C. Mol, W. F. Anderson, and M. Weigart. 1993. Residues that mediate DNA binding of autoimmune antibodies. J. Immunol. 150:4966-4977.

30. Manheimer-Lory, A., J. B. Katz, M. Pillinger, C. Ghossein, A. Smith, and B. Diamond. 1991. Molecular characteristics of antibodies bearing an anti-DNAassociated idiotype. J. Exp. Med. 174:1639-1652.

31. Seeman, N. C., J. M. Rosenberg, and A. Rich. 1976. Sequence specific recognition of double helical nucleic acids by proteins. Proc. Natl. Acad. Sci. USA. 73:804-808.

32. Diamond, B., J. B. Katz, E. Paul, C. Aranow, D. Lustgartenand, and M. D. Scharff. 1992. The role of somatic mutation in the anti-DNA response. Ann. Rev. Immunol. 10:731-757.

33. Agnello, V., A. Arbetter, G. I. DeKarsep, R. Powell, E. M. Tan, and F Joslin. 1980. Evidence for a subset of rheumatoid factors that cross-react with DNA-histone and have a distinct cross-idiotype. J. Exp. Med. 151:1514-1527.

34. Udey, J. A., and B. Blomberg. 1987. Human $\lambda$ light chain locus: organization and DNA sequences of three genomic $\mathrm{J}$ regions. Immunogenetics. 25:63-70. 